\title{
A constant approximation algorithm for the densest $k$-subgraph problem on chordal graphs
}

\author{
Maria Liazi ${ }^{a}, *, 1$, Ioannis Milis ${ }^{b}$, Vassilis Zissimopoulos ${ }^{\text {a }}$ \\ ${ }^{a}$ Department of Informatics and Telecommunications, \\ University of Athens, 15784 Athens, Greece \\ ${ }^{\mathrm{b}}$ Department of Informatics, \\ Athens University of Economics and Business, 10434 Athens, Greece
}

\begin{abstract}
The Densest $k$-Subgraph $(\mathrm{D} k \mathrm{~S})$ problem asks for a $k$-vertex subgraph of a given graph with the maximum number of edges. The $\mathrm{D} k \mathrm{~S}$ problem is NP-hard even for special graph classes including bipartite, planar, comparability and chordal graphs, while no constant approximation algorithm is known for any of these classes. In this paper we present a 3-approximation algorithm for the class of chordal graphs. The analysis of our algorithm is based on a graph theoretic lemma of independent interest.
\end{abstract}

Key words: Densest $k$-subgraph, Chordal graphs, Approximation algorithm

\section{Introduction}

In the Densest $k$-subgraph $(\mathrm{D} k \mathrm{~S})$ problem we are given a graph $G=(V, E)$, $|V|=n$, and an integer $k \leq n$, and we ask for a subgraph of $G$ induced by exactly $k$ of its vertices such that the number of edges of this subgraph is maximized. The problem is directly NP-hard as a generalization of the well known Maximum Clique problem. In the weighted version of the $\mathrm{D} k \mathrm{~S}$ we are

* Corresponding author.

Email addresses: mliazi@di.uoa.gr (Maria Liazi), milis@aueb.gr (Ioannis Milis), vassilis@di.uoa.gr (Vassilis Zissimopoulos).

1 The project is co-financed within Op. Education by the ESF (European Social Fund) and National Resources. 
also given non negative weights on the edges of $G$ and the goal is to find a $k$-vertex induced subgraph of maximum total edge weight.

During last years a large body of work has been concentrated on the design of approximation algorithms for both the $\mathrm{D} k \mathrm{~S}$ problem and its weighted version, based on a variety of techniques including greedy algorithms, LP relaxations and semidefinite programming. For a brief presentation of this body of work the reader is referred to [3] and the references therein. The best known approximation ratio for the $\mathrm{D} k \mathrm{~S}$ problem, which performs well for all values of $k$, is $O\left(n^{\delta}\right)$, for some $\delta<\frac{1}{3}[6]$, while a simple greedy algorithm in [2] achieves an approximation ratio of $O\left(\frac{n}{k}\right)$ even for the weighted version of the problem. On the other hand, it has been shown that the $\mathrm{D} k \mathrm{~S}$ problem does not admit a polynomial time approximation scheme (PTAS) [13]. However, there is not a negative result that achieving an approximation ratio of $O\left(n^{\epsilon}\right)$, for some $\epsilon>0$, is NP-hard. Concerning approximation algorithms for special cases of the problem it is known that the D $k \mathrm{~S}$ problem admits a PTAS for graphs of minimum degree $\Omega(n)$ as well as for dense graphs (of $\Omega\left(n^{2}\right)$ edges) when $k$ is $\Omega(n)$ [1]. Moreover, algorithms achieving approximation factors of 4 [17] and 2 [11] have been proposed for the weighted $\mathrm{D} k \mathrm{~S}$ problem on complete graphs where the weights satisfy the triangle inequality.

The $\mathrm{D} k \mathrm{~S}$ problem is trivial on trees (any subtree of $k$ vertices contains exactly $k-1$ edges). It is also known that $\mathrm{D} k \mathrm{~S}$ is polynomial for graphs of maximal degree two [7] as well as for cographs, split graphs and k-trees [4]. On the other hand the DkS problem remains NP-hard for bipartite graphs [4], even of maximum degree three [7], as well as for comparability graphs, chordal graphs [4] and planar graphs [12]. The weighted version of the D $k \mathrm{~S}$ problem is polynomial on trees either if we ask for a connected solution $[10,14,15]$ or for a disconnected one [16]. In fact, the result for the latter case is implied by a result for the solution of the quadratic 0-1 knapsack problem on edge series-parallel graphs in [16].

In the next section we introduce the reader to the class of chordal graphs and their properties and we give our notation. In Section 3 we present the approximation algorithm and the lemmas yielding our approximation ratio. We conclude in Section 4.

\section{Definitions and Notation}

A clique of an undirected graph, $G=(V, E)$, is a subset of its vertices, $C \subseteq V$, inducing a complete subgraph in $G$. The size $|C|$ of a clique is the number of its vertices. A maximal clique is a clique, which is not contained in any larger clique. A largest maximal clique is called maximum clique. A vertex of a graph 
$G$ is called simplicial if its adjacent vertices induce a complete subgraph in $G$. An order $\left\langle u_{1}, u_{2}, \ldots, u_{n}\right\rangle$ of the vertices of $G$, is called perfect elimination order if each $u_{i}$ is a simplicial vertex of the subgraph of $G$ induced by the vertices $\left\{u_{i}, u_{i+1}, \ldots, u_{n}\right\}$.

A graph is called chordal if every cycle of length strictly greater than three possesses a chord, that is, an edge joining two nonconsecutive vertices of the cycle. In the rest of this paper by $G=(V, E)$ we denote a chordal graph. It is well known that for a chordal graph, $G=(V, E)$, the following hold:

(i) $G$ has at most $m \leq|V|$ maximal cliques, $\mathcal{C}=\left\{C_{1}, C_{2}, \ldots, C_{m}\right\}$, which can be found in polynomial time [9].

(ii) $G$ has a simplicial vertex. Actually, if $G$ is not a clique, then it has two nonadjacent simplicial vertices [5].

(iii) $G$ has a perfect elimination order. Moreover, any simplicial vertex can start such an order [8].

By $G_{A}$ we denote the subgraph of $G$ induced by a subset $A \subseteq V$ of its vertices and by $G^{F}$ we denote the subgraph of $G$ induced by a subset $F \subseteq E$ of its edges. A direct consequence of the definition of the class of chordal graphs is that being chordal is a hereditary property inherited by every vertex-induced subgraph $G_{A}$ of $G$, but not by every edge-induced subgraph $G^{F}$ of $G$. It is also obvious that for every maximal clique $C_{i}$ of a vertex-induced or an edgeinduced subgraph of $G$, there is at least one maximal clique $C_{j}$ of $G$ such that $C_{i} \subseteq C_{j}$.

By $E(A)$ we denote the set of edges in a subgraph $G_{A}$ of $G$, while by $E(A, B)$ we denote the set of edges between two disjoint subsets $A, B \subseteq V$ of vertices of $G$ i.e., the set of edges with one of their endpoints in $A$ and the other in $B$.

By $S$ we denote a solution to the DkS problem, that is a subset $S \subseteq V$ such that $|S|=k$, while by $S^{*}$ we denote an optimal solution, that is a solution $S$ for which $|E(S)|$ is maximized.

Finally, we assume that $k>\left|C_{i}\right|, 1 \leq i \leq m$, for otherwise $S^{*}$ consists of any subset of $k$ vertices of some clique for which $\left|C_{i}\right| \geq k$.

\section{An approximation algorithm for the DkS problem on chordal graphs}

Since all the maximal cliques of a chordal graph $G=(V, E)$ can be found in polynomial time it is natural to study the $\mathrm{D} k \mathrm{~S}$ problem on those maximal cliques instead on $G$ itself. In this section we analyze the following simple greedy algorithm for finding an approximate solution to the $\mathrm{D} k \mathrm{~S}$ problem on 
a chordal graph $G$.

\section{Greedy Algorithm:}

1. Let $C_{1}, C_{2}, \ldots C_{m}$ be the maximal cliques of $G$, sorted in non-increasing order of their sizes.

2. Find the largest integer $t$ such that $k>\left|\bigcup_{i=1}^{t-1} C_{i}\right|=k^{\prime}$.

3. Return the solution $S$ consisting of all the vertices of the cliques $C_{1}, C_{2}, \ldots, C_{t-1}$ plus $k-k^{\prime}>0$ vertices of clique $C_{t}$.

The size of the maximal clique $C_{t}$ plays a crucial role in our analysis and it will be denoted by $L=\left|C_{t}\right|$.

We first obtain a lower bound on the number of edges $|E(S)|$ in the solution $S$ derived by the Greedy Algorithm for a chordal graph $G$. This bound is obtained by relating the solution $S$ to the solution that the Greedy Algorithm returns for a graph consisting of independent cliques of size $L$. Formally, for a chordal graph $G$ and the parameter $L$ defined above we consider the chordal graph $\widetilde{G}$ consisting of at least $\lceil k / L\rceil$ independent cliques all of size $L$.

Lemma 1 Let $S$ and $\widetilde{S}$ be the solutions that the Greedy Algorithm returns for the DkS problem on graphs $G$ and $\widetilde{G}$, respectively. It holds that $|E(S)| \geq$ $|E(\widetilde{S})|=\frac{k(L-1)-b(L-b)}{2}$, where $b=k \bmod L$.

Proof: To prove the bound of the lemma we consider also the solutions $S^{\prime}$ and $\widetilde{S}^{\prime}$ that the Greedy Algorithm returns for the Densest $k^{\prime}$-Subgraph (D $\left.k^{\prime} \mathrm{S}\right)$ problem on graphs $G$ and $\widetilde{G}$, respectively. Recall that $k^{\prime}=\left|\bigcup_{i=1}^{t-1} C_{i}\right|$.

Consider first the solutions $S$ and $S^{\prime}$. The solution $S$ consists of the $t-1$ largest maximal cliques of $G$, of $k^{\prime}$ vertices, plus a set $A \subseteq C_{t}$, of $a=k-k^{\prime}$ vertices, such that $\bigcup_{i=1}^{t-1} C_{i} \cap A=\emptyset$. Obviously, $0<a \leq\left|C_{t}\right|=L$. Moreover, $S \backslash S^{\prime}=A$ and therefore,

$$
|E(S)| \geq\left|E\left(S^{\prime}\right)\right|+\frac{a(a-1)}{2} .
$$

Consider next the solutions $S^{\prime}$ and $\widetilde{S}^{\prime}$. The solution $S^{\prime}$ consists of exactly the $t-1$ largest maximal cliques of $G$, of $k^{\prime}$ vertices. As all the maximal cliques of $S^{\prime}$ are of size at least $L$, it follows that all the vertices in $S^{\prime}$ have degree at least $L-1$. The solution $\widetilde{S}^{\prime}$ consists of $q^{\prime}=\left\lfloor k^{\prime} / L\right\rfloor$ independent cliques of size $L$ and one more independent clique, $B^{\prime}$, of size $b^{\prime}=k^{\prime} \bmod L$, that is $\left|\widetilde{S}^{\prime}\right|=q^{\prime} L+b^{\prime}$. Each one of the $b^{\prime}$ vertices in the clique $B^{\prime}$ of $\widetilde{S}^{\prime}$ has degree $b^{\prime}-1$. Hence, the solution $S^{\prime}$ contains at least $b^{\prime}\left(L-1-\left(b^{\prime}-1\right)\right)=b^{\prime}\left(L-b^{\prime}\right)$ more edges than the solution $\widetilde{S}^{\prime}$, that is $\left|E\left(S^{\prime}\right)\right| \geq\left|E\left(\widetilde{S}^{\prime}\right)\right|+b^{\prime}\left(L-b^{\prime}\right)$. Therefore,

$$
|E(S)| \geq\left|E\left(\widetilde{S}^{\prime}\right)\right|+b^{\prime}\left(L-b^{\prime}\right)+\frac{a(a-1)}{2} .
$$

Consider finally the solutions $\widetilde{S}^{\prime}$ and $\widetilde{S}$. The solution $\widetilde{S}$ consists of $q=\lfloor k / L\rfloor$ 
independent cliques of size $L$ and one more independent clique, $B$, of size $b=k \bmod L$, that is $|\widetilde{S}|=q L+b$. Moreover, $|\widetilde{S}|-\left|\widetilde{S}^{\prime}\right|=a$.

If $a \leq b$, then the solution $\widetilde{S}^{\prime}$ can be obtained from the $\widetilde{S}$, by removing $a$ vertices of the clique $B$ of $\widetilde{S}$. In this case $\left|\widetilde{S}^{\prime}\right|=q L+b^{\prime}$, where $b^{\prime}=b-a$, and $|E(\widetilde{S})|=\left|E\left(\widetilde{S}^{\prime}\right)\right|+\frac{a(a-1)}{2}+b^{\prime} a$. Therefore,

$$
|E(S)| \geq|E(\widetilde{S})|-b^{\prime} a+b^{\prime}\left(L-b^{\prime}\right)=|E(\widetilde{S})|+b^{\prime}(L-b),
$$

and since $L>b$, the inequality of the lemma follows.

If $a>b$, then the solution $\widetilde{S}^{\prime}$ can be obtained from the $\widetilde{S}$, by removing all the $b$ vertices of the clique $B$ and $x=a-b$ vertices of the $q-t h$ clique of size $L$ of $\widetilde{S}$. In this case $\left|\widetilde{S}^{\prime}\right|=(q-1) L+b^{\prime}$, where $a-b=L-b^{\prime}$, and $|E(\widetilde{S})|=\left|E\left(\widetilde{S}^{\prime}\right)\right|+\frac{b(b-1)}{2}+\frac{x(x-1)}{2}+x b^{\prime}=\left|E\left(\widetilde{S}^{\prime}\right)\right|+\frac{a(a-1)}{2}-x b+x b^{\prime}=$ $\left|E\left(\widetilde{S}^{\prime}\right)\right|+\frac{a(a-1)}{2}+(a-b)\left(b^{\prime}-b\right)$. Therefore,

$$
|E(S)| \geq|E(\widetilde{S})|-(a-b)\left(b^{\prime}-b\right)+b^{\prime}\left(L-b^{\prime}\right)=|E(\widetilde{S})|+\left(L-b^{\prime}\right) b,
$$

and since $L>b^{\prime}$ the inequality of the lemma follows.

The solution $\widetilde{S}$ consists of $\frac{k-b}{L}$ full cliques plus $b<L$ vertices from the clique $B$. Hence, the number of edges in $\widetilde{S}$ is

$$
|E(\widetilde{S})|=\frac{k-b}{L} \cdot \frac{L(L-1)}{2}+\frac{b(b-1)}{2}=\frac{k(L-1)-b(L-b)}{2} .
$$

Next lemma, which is of independent interest, gives un upper bound on the number of edges of a chordal graph as a function of the size of its maximum clique.

Lemma 2 Let $c \geq 2$ be the size of a maximum clique of a chordal graph $G=(V, E)$. It holds that $|E| \leq(c-1)\left(|V|-\frac{c}{2}\right)$ and this bound is the best possible.

Proof: The graph $G$, as a chordal one, has a perfect elimination order. We remove from $G$ vertices (and their incident edges) in a perfect elimination order until the remaining number of its vertices is $c$.

Since the size of a maximum clique of $G$ is $c$, each removed vertex has degree at most $c-1$. Thus, the number of the edges removed during this process, let $\left|E_{1}\right|$, is at most $(|V|-c)(c-1)$. Moreover, the remaining number of edges, let $\left|E_{2}\right|$, is at most $\frac{c(c-1)}{2}$ (when the remaining $c$ vertices form a clique).

Therefore, it follows that

$$
|E|=\left|E_{1}\right|+\left|E_{2}\right| \leq(|V|-c)(c-1)+\frac{c(c-1)}{2}=(c-1)\left(|V|-\frac{c}{2}\right) .
$$

To prove that this bound is the best possible consider the chordal graph $G=(V, E)$ consisting of a clique, $C$, of size $c-1$ and $|V|-c+1$ independent vertices each one of them adjacent to all vertices of $C$. Observe that a maximum clique of $G$ consists of the clique $C$ plus one of the independent vertices, and it 
is of size $c$. For this graph $G$ it holds that $|E|=\frac{(c-1)(c-2)}{2}+(|V|-c+1)(c-1)=$ $(c-1)\left(|V|-\frac{c}{2}\right)$. Note that if $c=|V|$, then $G$ becomes a complete graph.

Let us now relate the solution $S$ of the Greedy Algorithm to an optimal solution $S^{*}$ to the D $k \mathrm{~S}$ problem on a chordal graph $G$. Let $S^{*}=A \cup B$, where $A=S^{*} \cap S$ is the subset of vertices of $S^{*}$ that belong to $S$ and $B=S^{*} \backslash A$ is the subset of vertices of $S^{*}$ that do not belong to $S$. Let also $\Gamma \subseteq A$ be the subset of vertices in $A$ that have adjacent vertices in $B$ and $F=E(B) \cup E(\Gamma, B)$. Obviously, $\Gamma \cap B=\emptyset$ and $\left|E\left(S^{*}\right)\right|=|E(A)|+|E(B)|+|E(\Gamma, B)|=|E(A)|+|F|$.

In order to bound the number of edges in an optimal solution $S^{*}$ we shall consider the edge-induced subgraph $G^{F}=(\Gamma \cup B, F)$ as well as the vertexinduced subgraph $G_{B \cup \Gamma}$ of $G$. Note that $G_{B \cup \Gamma}$, as a vertex-induced subgraph of $G$, is a chordal graph, while $G^{F}$, as an edge-induced subgraph of $G$, is in general a non chordal one. Next proposition gives a useful structural property of the subgraph $G^{F}$.

Proposition 1 All the maximal cliques of the graph $G^{F}=(\Gamma \cup B, F)$ are of size at most $L$.

Proof: The solution $S$ of the Greedy Algorithm contains vertices from maximal cliques of $G$ of size at least $L$ and the vertices in $B$ do not belong to $S$. Therefore, the vertices in $B$ belong to maximal cliques of $G$ of size at most $L$.

Consider first the set $B$ of vertices of the subgraph $G^{F}$. Since they belong to maximal cliques of $G$ of size at most $L$, they also form in $G^{F}$ maximal cliques of size at most $L$.

Consider next the set $\Gamma$ of vertices of the subgraph $G^{F}$. By the definition of the subgraph $G^{F}$, it follows that: a) they are independent in $G^{F}$, and b) every one of them has at least one adjacent vertex in $B$. Assume that such vertex belongs to a maximal clique, $K$, of $G^{F}$ of size greater than $L$. As the vertices of $\Gamma$ are independent in $G^{F}$, it follows that at least one vertex of $B$ belongs also in such a clique $K$ of $G^{F}$, and, therefore, to a maximal clique of $G$ of size greater than $L$, a contradiction to the definition of the set $B$. Hence the vertices of $\Gamma$ belong to maximal cliques of $G^{F}$ of size at most $L$.

Therefore, all maximal cliques of the graph $G^{F}$ are of size at most $L$.

Using Proposition 1 we can now prove that the bound of Lemma 2 holds also for the graph $G^{F}$.

Lemma 3 For the edge-induced graph $G^{F}=(\Gamma \cup B, F)$ of $G$ it holds that $|F| \leq(L-1)\left(|\Gamma \cup B|-\frac{L}{2}\right)$.

\section{Proof:}


We work analogously as in Lemma 2 . The graph $G_{B \cup \Gamma}$, as a vertex-induced subgraph of $G$, is a chordal one and it has a perfect elimination order. Following this order (of the graph $G_{B \cup \Gamma}$ ), we remove vertices from the graph $G^{F}$ until the remaining number of its vertices is $L$.

By Proposition 1 the size of a maximum clique of $G^{F}$ is $L$ and, hence, the degree, in $G^{F}$, of each removed vertex is at most $L-1$. Thus, the number of the removed edges of $G^{F}$ during this process, let $\left|E_{1}\right|$, is at most $(|B \cup \Gamma|-L)(L-1)$. Moreover, the remaining edges of $G^{F}$, let $\left|E_{2}\right|$, is at most $\frac{L(L-1)}{2}$ (when the remaining $L$ vertices form a clique).

Therefore, it follows that

$|E|=\left|E_{1}\right|+\left|E_{2}\right| \leq(|B \cup \Gamma|-L)(L-1)+\frac{L(L-1)}{2}=(L-1)\left(|B \cup \Gamma|-\frac{L}{2}\right)$.

Applying Lemma 3 on $G^{F}$, (with $|\Gamma \cup B| \leq\left|S^{*}\right|=k$ ) we obtain

$$
|F|=|E(B)|+|E(\Gamma, B)| \leq(L-1)\left(k-\frac{L}{2}\right) .
$$

For the edges $E(A)$ it holds that $|E(A)| \leq|E(S)|$, since $A \subseteq S$. We also know, by Lemma 1 , that

$$
|E(S)| \geq \frac{k(L-1)-b(L-b)}{2} .
$$

Therefore,

$$
\frac{\left|E\left(S^{*}\right)\right|}{|E(S)|}=\frac{|E(A)|+|F|}{|E(S)|} \leq 1+\frac{|F|}{|E(S)|} \leq 1+\frac{(L-1)(2 k-L)}{k(L-1)-b(L-b)}
$$

By recalling that $b=k \bmod L \leq L-1$ and by distinguishing between two cases for $b(b \leq L / 2$ and $b>L / 2)$ it is easy to prove that $\frac{(L-1)(2 k-L)}{k(L-1)-b(L-b)} \leq 2$. Thus the next theorem follows.

Theorem 1 There is a 3-approximation algorithm for the DkS problem on chordal graphs.

\section{Concluding remarks}

We have shown a 3-approximation algorithm for the $\mathrm{D} k \mathrm{~S}$ problem on chordal graphs, which, up to our knowledge, is the first constant approximation algorithm for an NP-hard variant of the problem on non-dense graphs. Concerning 
the tightness of our analysis we succeeded to construct counterexamples for which our algorithm gives a solution of at least half of the edges of an optimal one.

On the other hand many questions concerning the frontier between hard and polynomial solvable or approximable, within a constant ratio, variants of the $\mathrm{D} k \mathrm{~S}$ problem, remain open. Such an outstanding open question concerns the complexity of the $\mathrm{D} k \mathrm{~S}$ problem on interval graphs or even on proper interval graphs. The existence of a constant approximation algorithm for the NPhard variant of the $\mathrm{D} k \mathrm{~S}$ problem on planar graphs is another interesting open question.

Acknowledgment. The crucial suggestions of an anonymous referee for the simplification of the proofs of Lemmas 2 and 3 are gratefully acknowledged.

\section{References}

[1] S. Arora, D. Karger, and M. Karpinski. Polynomial time approximation schemes for dense instances of NP-hard problems. In Proceedings of the 27th annual ACM symposium on Theory of Computing, pages 284-293, 1995.

[2] Y. Asahiro, K. Iwama, H. Tamaki, and T. Tokuyama. Greedily finding a dense subgraph. Journal of Algorithms, 34(2):203-221, 2000.

[3] A. Billionnet and F. Roupin. A deterministic algorithm for the densest k-subgraph problem using linear programming. Technical Report No486, CEDRIC, CNAM-IIE, Paris, 2004.

[4] D. G. Corneil and Y. Perl. Clustering and domination in perfect graphs. Discrete Applied Mathematics, 9:27-39, 1984.

[5] G. A. Dirac. On rigid circuit graphs. Abhandlungen aus dem Mathematischen Seminar der Universität Hamburg, 25:71-76, 1961.

[6] U. Feige, G. Kortsarz, and D. Peleg. The dense k-subgraph problem. Algorithmica, 29(3):410-421, 2001.

[7] U. Feige and M. Seltser. On the densest k-subgraph problem. Technical Report CS97-16, Weizmann Institute, 1997.

[8] D. R. Fulkerson and O. A. Gross. Incidence matrices and interval graphs. Pacific Journal of Mathematics, 15:835-855, 1965.

[9] F. Gavril. Algorithms for minimum coloring, maximum clique, minimum covering by cliques and maximum independent set of chordal graph. SIAM Journal on Computing, 1(2):180-187, 1972. 
[10] O. Goldschmidt and D. Hochbaum. k-edge subgraph problems. Discrete Applied Mathematics and Combinatorial Operations Research and Computer Science, 74(2):159-169, 1997.

[11] R. Hassin, S. Rubinstein, and A. Tamir. Approximation algorithms for maximum dispersion. Operations Research Letters, 21(3):133-137, 1997.

[12] J. M. Keil and T. B. Brecht. The complexity of clustering in planar graphs. Journal of Combinatorial Mathematics and Combinatorial Computing, 9:155159, 1991.

[13] S. Khot. Ruling out PTAS for graph min-bisection, densest subgraph and bipartite clique. In Proceedings of the 45th Annual IEEE Symposium on Foundations of Computer Science, pages 136-145, 2004.

[14] F. Maffioli. Finding a best subtree of a tree. Technical Report 91.041, Politechnico di Milano, Dipartimento di Elektronica, 1991.

[15] Y. Perl and Y. Shiloach. Efficient optimization of monotonic functions on trees. SIAM Journal of Algebric and Discrete Methods, 4(4):512-516, 1983.

[16] D. J. Jr. Rader and G. J. Woeginger. The quadratic 0-1 knapsack problem with series-parallel support. Operation Research Letters, 30(3):159-166, 2002.

[17] S. S. Ravi, D. J. Rosenkrantz, and G. K. Tayi. Heuristic and special case algorithms for dispersion problems. Operations Research, 42(2):299-310, 1994. 